
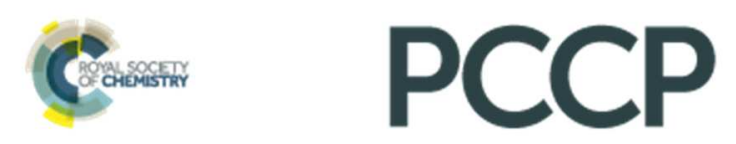

\title{
Dynamic viscosity mapping of the oxidation of squalene aerosol particles
}

\begin{tabular}{|r|l|}
\hline Journal: & Physical Chemistry Chemical Physics \\
\hline Manuscript ID & CP-ART-08-2016-005674.R1 \\
\hline Article Type: & Paper \\
\hline Date Submitted by the Author: & 05-Oct-2016 \\
\hline Complete List of Authors: & $\begin{array}{l}\text { Athanasiadis, Thanos; Imperial College London, Chemistry } \\
\text { Fitzgerald, Clare; University of Cambridge, Cavendish Laboratory } \\
\text { Davidson, Nick; University of Birmingham, } \\
\text { Giorio, Chiara; University of Cambridge } \\
\text { Botchway, Stanley; STFC, ; } \\
\text { Ward, Andrew; Rutherford Appleton Laboratory, Lasers for Science Facility } \\
\text { Kalberer, Markus; University of Cambridge, Department of Chemistry } \\
\text { Pope, Francis D.; University of Birmingham, School of Geography, Earth } \\
\text { and Environmental Science } \\
\text { Kuimova, Marina; Imperial College London, Chemistry }\end{array}$ \\
\hline
\end{tabular}




\title{
Journal Name
}

\section{ARTICLE}

\section{Dynamic viscosity mapping of the oxidation of squalene aerosol particles.}

Received 00th January 20xx, Accepted 00th January 20xx DOI: $10.1039 / x 0 \times x 00000 x$

\section{www.rsc.org/}

\begin{abstract}
Athanasios Athanasiadis, ${ }^{\mathrm{a}}+$ Clare Fitzgerald,${ }^{\mathrm{b}}+$ Nicholas M. Davidson, ${ }^{\mathrm{c}}$ Chiara Giorio, ${ }^{\mathrm{b}}$ Stanley W. Botchway, ${ }^{d}$ Andrew D. Ward, ${ }^{d}$ Markus Kalberer, ${ }^{\text {b* }}$ Francis D. Pope ${ }^{c *}$ and Marina K. Kuimova ${ }^{a *}$
\end{abstract}

\begin{abstract}
Organic aerosols (OA) play important roles in multiple atmospheric processes, including climate change, and can impact on human health. The physico-chemical properties of $\mathrm{OA}$ are important for all these processes and can evolve through reactions with various atmospheric components, including oxidants. The dynamic nature of these reactions makes it challenging to obtain a true representation of their composition and surface chemistry. Here we investigate the microscopic viscosity of model OA composed of squalene, undergoing chemical aging. We employ Fluorescent Lifetime Imaging Microscopy (FLIM) in conjunction with viscosity sensitive probes termed molecular rotors, in order to image changes in microviscosity in real time during oxidation with ozone and hydroxyl radicals, which are two key oxidising species in the troposphere. We also recorded Raman spectra of levitated particles to follow the reactivity during particle ozonolysis. The levitation of droplets was achieved via optical trapping that enabled simultaneous levitation and measurement via FLIM or Raman spectroscopy and allowed the true aerosol phase to be probed. Our data revealed a very significant increase in viscosity of levitated squalene droplets upon ozonolysis, following their transformation from liquid to solid phase that was not observable when the oxidation was carried out on coverslip mounted droplets. FLIM imaging with sub-micron spatial resolution also revealed spatial heterogeneity in viscosity distribution of oxidised droplets. Overall, a combination of molecular rotors, FLIM and optical trapping is able to provide powerful insights into OA chemistry and the microscopic structure that enables the dynamic monitoring of microscopic viscosity in aerosol particles in its true phase.
\end{abstract}

\section{Introduction}

Aerosols play a prominent role in atmospheric chemistry, cloud formation, biogeochemical cycles and radiative forcing. ${ }^{1}$ They can serve as atmospheric cloud condensation nuclei and ice nuclei ${ }^{2}$. Moreover, aerosols have been linked to air pollution related illnesses like asthma, inflammation of the respiratory system, cancer, oxidative stress, heart attacks and stroke. $^{2-4}$ Thus it is imperative to increase our understanding of their physical and chemical properties to better monitor their interactions with the environment and living organisms. However, due to a wide variability in aerosol size and composition, there is a major uncertainty in modelling and data interpretation related to aerosol physico-chemical

\footnotetext{
a. Department of Chemistry, Imperial College London, London, SW7 2AZ, UK. E-mail: m.kuimova@imperial.ac.UK

b. Department of Chemistry, University of Cambridge, Cambridge, CB2 1EW, UK.

E-mail: markus.kalberer@atm.ch.cam.ac.UK

School of Geography, Earth and Environmental Science, University of

Birmingham, Edgbaston, B15 2TT, UK. E-mail: f.pope@bham.ac.UK

d. Central Laser Facility, Research Complex at Harwell, Rutherford Appleton

Laboratory, Chilton, Didcot, Oxon OX11 OQX, UK.

† These authors contributed equally to this work.

Electronic Supplementary Information (ESI) available: [details of any supplementary information available should be included here]. See DOI: $10.1039 / \mathrm{x} 0 \mathrm{xx} 00000 \mathrm{x}$
}

properties.

Aerosol particle viscosity and phase is highly important since it can determine the ability of chemical species such as oxidants, water or organic matter to diffuse into the particle bulk $^{5}$. An aerosol's viscous state also has direct implications on the particle morphology, phase behaviour, optical properties, aging processes and lifetime. At the same time, only few direct measurement methods are available for monitoring such properties ${ }^{1,6-16}$. Recently, we have demonstrated that viscosity of model organic aerosols, such as oleic acid droplets, and secondary organic aerosols (SOA) produced from the oxidation of myrcene or $\alpha$-pinene can be measured and imaged in a spatially and time-resolved manner using Fluorescence Lifetime Imaging Microscopy (FLIM) of molecular rotors ${ }^{17}$. In our earlier study, ${ }^{17}$ the droplets were deposited onto a glass coverslip for FLIM measurements. More recently we have combined FLIM of incorporated molecular rotors with optical trapping of pharmaceutical aerosol droplets in order to directly measure the viscosity of aerosols, avoiding contact with surfaces. ${ }^{18}$ Here we extend this technique to directly monitor the dynamics of oxidation of levitated organic squalene aerosol, using two key oxidising species common in the troposphere: ozone and the hydroxyl $(\mathrm{OH})$ radical.

Molecular rotors are viscosity sensitive fluorophores, whose fluorescence lifetime and intensity are dependent on 
the microviscosity of their immediate surroundings. Since fluorescence lifetime is a concentration-independent parameter that can be imaged in a spatially-resolved manner using FLIM, lifetime mapping of microscopic objects incorporating molecular rotors provides a versatile method that can provide accurate, real-time measurements from different areas of an organic aerosol droplet. ${ }^{19}$

In our recent study we have measured microscopic viscosity of secondary organic aerosols of myrcene and $\alpha$ pinene during atmospherically relevant changes in relative humidity $(\mathrm{RH})$. Likewise, we were able to study oxidative ageing of oleic acid droplets upon exposure to ozone. Our method is capable of providing diffraction-limited spatial and temporal resolution in viscosity determination that cannot be easily matched by other currently available methods.

One of the limitations of our previous study by Hosny et al has been the use of droplets suspended on the microscope coverslip as model aerosol particles ${ }^{17,20}$. Since aerosol is a multiphase system composed of both a gas and a condensed phase, its physico-chemical properties including viscosity, can depend on the composition, state of matter, size, morphology, temperature and pressure of each of these phases. Fitzgerald et $a^{118}$ extended this work by combining the FLIM viscosity measurements with optical trapping of model aerosol to better simulate the aerosol in their natural environment by removing contact with a coverslip. This work focused on measuring $\mathrm{RH}$ dependent viscosities of the suspended aerosol. This unique setup comprised of an environmental chamber where humidity and temperature as well as gas flow can be precisely controlled, a FLIM microscope and an optical trapping system, which allowed us to examine a single levitated particle reacting for several hours as its gas phase composition and physical conditions changed.

In the present work we utilise a hydrophobic molecular rotor Bodipy- $\mathrm{C}_{10}$ incorporated directly into aerosol droplets in conjunction with optical tweezers to monitor the real time microviscosity changes of organic aerosol during aging. We compare these results with measurements of aerosol aging on the flat surface of a coverslip.

Terpenes are among the most frequently occurring type of volatile organic compounds (VOCs) in the atmosphere ${ }^{21,22}$. The atmospheric oxidation of these VOCs results in lower volatility products which can form OA. Squalene is a 30-carbon naturally occurring triterpene (Figure S1, ESI) which herein we use as a model organic aerosol. It has a vital role in the synthesis of sterols in animals and plants ${ }^{23,24}$ and is also produced by human skin ${ }^{25,26}$ and found as an ingredient in many household oils, used for cooking and frying ${ }^{27}$. Due to skin shedding (desquamation), squalene containing skin flakes are likely a major constituent of indoor dust and hence aerosol. ${ }^{28}$ Therefore it is found in detectable concentrations within aerosol in cities and urban areas. ${ }^{29,30}$ In the presence of strong oxidants such as ozone, squalene yields high molecular weight products such as polyunsaturated aldehydes, similar to those found in the very complex naturally occurring secondary organic aerosols (SOA) in the atmosphere ${ }^{27,31}$. The initial step of squalene ozonolysis will be via the well-known Criegee mechanism which generates carbonyls and carbonyl oxides also known as "Criegee intermediates" via a primary ozonide. ${ }^{32}$ Known squalene ozonolysis products contain many functional groups, such as ketones, alcohols, hydroperoxides and carboxylic acids also found in SOA in the atmosphere $26,27,33$. A high resolution mass spectrometry study showed that over 1300 products are generated from squalene ozonolysis and the accompanying model predicts many more are possible which indicates the complexity of the system. ${ }^{34}$

Here we studied how the viscosity of levitated squalene droplets changes under oxidation by ozone and hydroxyl radicals, two common atmospheric oxidants.

\section{Results and Discussion}

A molecular rotor Bodipy- $\mathrm{C}_{10}$ that was previously well characterised for viscosity measurements in oleic acid droplets $^{17,20}$, as well as in lipid vesicles ${ }^{35-37}$, was used for viscosity measurements. Bodipy- $\mathrm{C}_{10}$ is sensitive to a wide range of viscosities from 10 to $5000 \mathrm{mPa} \mathrm{s}$, corresponding to fluorescence lifetime changes from 1 up to ca. 6 ns (Figure S2, ESI), ${ }^{36}$ a range that is within Time Correlated Single Photon Counting FLIM instrument capabilities. Furthermore, fluorescence decays of Bodipy- $\mathrm{C}_{10}$ in homogeneous media can be fitted using monoexponential functions, allowing straightforward lifetime fitting.

In order to facilitate aerosol nebulisation and particle delivery to the environmental chamber, squalene was mixed with isopropanol at a ratio of $1: 15$. Bodipy- $C_{10}$ was added at a concentration of $20 \mu \mathrm{M}$. Once trapped, the volatile isopropanol evaporated leaving only squalene containing Bodipy- $\mathrm{C}_{10}$ trapped. The absence of isopropanol was confirmed by the fact that the lifetime of trapped squalene droplets matched the value from the bulk squalene measurements. In addition there was no evidence of isopropanol in the Raman spectra (see later discussion).

As a control, we have recorded brightfield fluorescence images of squalene droplets that were nebulised, trapped and left to equilibrate for 30 minutes in ambient conditions and subsequently carefully released onto the surface of the coverslip. Upon release all droplets spread upon the coverslip surface thus proving that they retained their liquid phase (Fig. $1 \mathrm{~A})$ and therefore were not adversely affected by trapping.

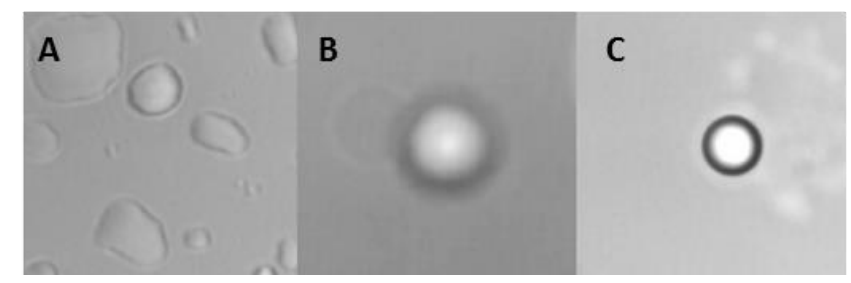

Figure 1: Brightfield microscopy images of a squalene droplet released from an optical trap onto the coverslip after (A) no ozone exposure, (B) exposure to ozone for $30 \mathrm{~min}$ at an ozone concentration of $53 \mathrm{ppm}$ (C) exposure to ozone for $75 \mathrm{~min}$ at an ozone concentration of $53 \mathrm{ppm}$, until no further changes in fluorescence lifetime of Bodipy$\mathrm{C}_{10}$ was visible. 

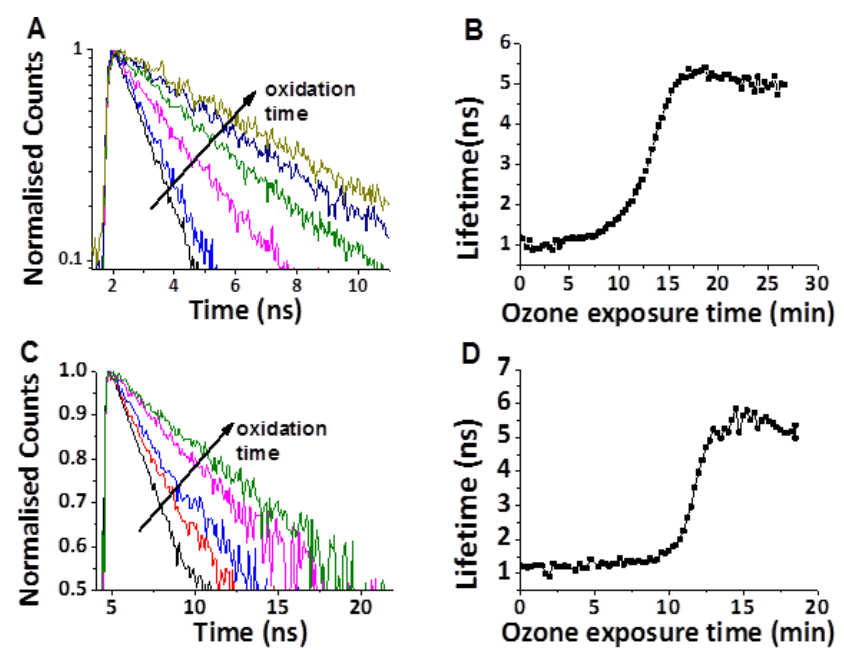

Figure 2: Decay traces of Bodipy- $\mathrm{C}_{10}$ recorded in (A) deposited and (C) trapped squalene droplets during oxidation with $53 \mathrm{ppm}$ of ozone for $20 \mathrm{~min}$. The traces in $\mathrm{A}$ and $C$ can be fitted using monoexponential function and the resulting evolution of the lifetime during oxidation is shown in (B) for deposited $(30 \mu \mathrm{m})$ and (D) trapped $(8 \mu \mathrm{m})$ droplets.

As a control, Bodipy- $\mathrm{C}_{10}$ was added to squalane droplets and exposed to ozone. Due to its saturated nature squalane did not react with ozone and no physical changes were observed regarding the droplet's viscosity or morphology (Figure S3, ESI). From this lack of lifetime change we can conclude that Bodipy- $C_{10}$ does not itself contribute to or interact with the processes of aerosol ozonolysis. Thus its ability to sense viscosity is not affected during ozonolysis.

The kinetics of viscosity changes due to ozonolysis was measured in trapped droplets and the results were compared to those in droplets resting on a coverslip surface, Figure 2. The collective decays from the whole volume of the droplet were recoded every 30 seconds, thus providing a good signal/noise, allowing good temporal resolution of the process. Before starting the ozonolysis, the trapped droplets were imaged in ambient conditions, with a flow of air on. The fluorescence decay of Bodipy- $\mathrm{C}_{10}$ from droplets at that stage was monoexponential and could be fitted with a lifetime of $c a$. $1.0 \mathrm{~ns}$, corresponding to a viscosity value of approximately $40 \mathrm{mPa}$ s. Once the ozone flow was initiated at $53 \mathrm{ppm}$, we observed an increase in the lifetime over time, up to the value of 5.5-6 ns, Figure 2C. The decays remained monoexponential until the fitted fluorescence lifetimes reached a maximum observed value of $6 \mathrm{~ns}$ within approximately 15 minutes at 53 ppm of ozone (Fig. 2D). The measurement continued until there was no further increase in lifetime.

In the case of the OA deposited on the coverslip, squalene was sprayed on the coverslip and sealed within the environmental chamber. A sealant gel was used to avoid gas leakage from the surface between the coverslip and the chamber. As for the trapped droplets, we observed monoexponential decays from all droplets, and the fitting of all traces produced a set of Bodipy- $\mathrm{C}_{10}$ lifetimes that increased in an S-shaped kinetics curve following droplet ozonolysis, Figure 2. The maximum lifetime (and hence the viscosity) value achieved at the end of ozonolysis, both in trapped and in coverslip deposited droplets, irrespective of their size, was similar, ca 5.0 - 6 ns.

It is noteworthy that the maximum value of the Bodipy- $\mathrm{C}_{10}$ lifetime observed in these experiments, ca $6 \mathrm{~ns}$, is at the limit of our calibration curve for this particular molecular rotor. In fact we have measured the fluorescence decay of Bodipy- $\mathrm{C}_{10}$ embedded in the glassy matrix of ethanol-methanol $\left(\eta=2 \times 10^{14}\right.$ $\mathrm{mPa} \mathrm{s})$ and 2-Methyltetrahydrofuran $\left(\eta=4 \times 10^{9} \mathrm{mPa} \mathrm{s}\right)$ at $77 \mathrm{~K}$ and obtained the values of 6 and $5.7 \mathrm{~ns}$, respectively, for their fluorescence lifetime (Figure S2, ESI). Thus, the limiting values measured for oxidised squalene droplets approach the high viscosity limit where the molecular rotor loses its sensitivity to increasing viscosity.

The Bodipy- $C_{10}$ calibration curve was measured earlier ${ }^{36,35}$ in methanol/glycerol mixtures of increasing viscosity and should conform to the Förster-Hoffmann equation ${ }^{19}$, namely

$$
\log \tau_{f}=\log \frac{z}{k_{r}}+\alpha \log \eta
$$

where $\tau_{\mathrm{f}}$ is the fluorescence lifetime, $\eta$ viscosity, $k_{\mathrm{r}}$ radiative decay rate and $\alpha, z$ constants.

This equation works well between 15 and $1000 \mathrm{mPa} \mathrm{s.}{ }^{37}$ However, at higher viscosity values, as the maximum quantum yield for the rotor is approached, the linearity between the $\log \tau_{f}$ and $\log \eta$ was lost (Figure S2, ESI) ${ }^{35,36}$. Thus, in the range of lifetimes exceeding $5 \mathrm{~ns}$, a very small change in fluorescence lifetime corresponds to a large change in viscosity. For fluorescence lifetime values approaching $6 \mathrm{~ns}$, the viscosity is hard to assign. While we nominally assign the viscosity of ca $5000 \mathrm{mPa} s$ to oxidised squalene droplets (that display lifetimes in the range 5-6 ns), both suspended and on the coverslip, we note that these lifetimes exceed the range where we can assign viscosity values with certainty. Therefore the real viscosity value achieved as a result of the oxidation may exceed $5000 \mathrm{mPa} s$ and can in fact be as high as $10^{14} \mathrm{mPa} \mathrm{s}$ (Figure S2).

The reason for the increase in viscosity is thought to be due to the appearance of squalene oxidation products. These products are highly oxidised species containing a mixture of carbonyl and carboxylic acid functional groups, see Figure S4 and Tables S1, S2, ESI. Secondary oxidation products can also form during the addition of first generation products with the Criegee Intermediate and each other. These molecules will be bulkier than the monomers and also oxidised, decreasing the general mobility within the oxidised mixture. Both factors (oxidation and oligomer formation) will have contributed to the measured increase in viscosity.

The phase of the oxidised droplets was further investigated by examining the morphology of the oxidised droplets during and at the end of ozonolysis, Figure 1. For this the trapped droplets were released from the trap after 30 and 75 minutes of exposure to $53 \mathrm{ppm}$ ozone. Once released, the droplet in Figure $1 \mathrm{~B}$ held its shape for a few seconds and then deformed slightly. During this deformation process the core of the droplet appeared to be in a more viscous state compared to 
the shell, since it was degrading slower, whereas the surface in a less viscous state moving faster towards the coverslip (Fig. 1B). When the experiment was repeated after 75 minutes of ozonolysis at $53 \mathrm{ppm}$, upon the release onto the coverslip the droplet retained its perfect spherical shape confirming that it has solidified (Figure $1 \mathrm{C}$ ), thus far exceeding nominal viscosity of $5000 \mathrm{mPa} s$ measured with the Bodipy- $\mathrm{C}_{10}$ molecular rotor. We note that the lower estimate of viscosity for a solid object is ca $10^{6} \mathrm{mPa} \mathrm{s},{ }^{38}$ beyond the measurement capability of the Bodipy- $\mathrm{C}_{10}$ rotor. We were not able to detect the glassy core in the coverslip deposited squalene droplets in the course of oxidation. The liquid phase of the droplets at the end of the oxidation was further confirmed by a 'scrape' test, Figure S5.

Having studied the kinetics of the droplet oxidation, we next attempted to image the distribution of viscosities within oxidised droplets. We have recorded a series of FLIM images of a $5 \mu \mathrm{m}$ trapped droplet containing Bodipy- $\mathrm{C}_{10}$ during the course of ozone oxidation. While images recorded at the start and at the early stages of ozonolysis showed a homogeneous distribution of lifetimes, the image showed interesting features once the ozonolysis was completed, Figure 3.

The Bodipy- $\mathrm{C}_{10}$ molecular rotor is characterised by monoexponential excited state decays, if it is placed in a homogeneous environment. Indeed, the FLIM images of the trapped squalene droplets recorded at early stages of ozonolysis can be fitted using monoexponential decay function with acceptable goodness of fit values $\left(\chi^{2}\right)$ (Figure S6, ESI). However, after the ozonolysis was complete, monoexponential fitting analysis indicated certain areas in the centre of the droplet where the goodness of the fit was not satisfactory, $\chi^{2}$ $>2.5$ (Figure 3A). Further analysis revealed that the decay traces of the individual pixels in the centre of the droplets had a prominent scatter peak (Figure 3B). This scattering peak appeared gradually as the ozonolysis progressed and dominated the decays after about 15 min ozone exposure, Figure $3 C$. At the same time the longer lifetime component (attributed to the emission of Bodipy- $\mathrm{C}_{10}$ itself) remained uniform throughout the particle, Figure 3. We attributed the appearance of the scattering peak to the formation of a glassy and/or crystalline core in the centre of the droplet, which can efficiently scatter excitation light. The peak became more prominent in the course of the ozonolysis due to the bleaching of Bodipy- $\mathrm{C}_{10}$ by ozone and the excitation laser used for imaging. We observed no scattering peak and no evidence of droplet solidification when droplets deposited directly on the coverslip were measured.

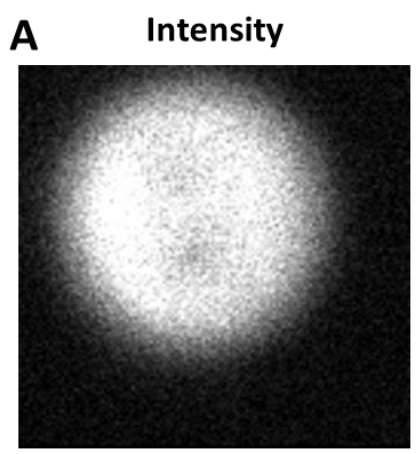

\section{Lifetime with zero}

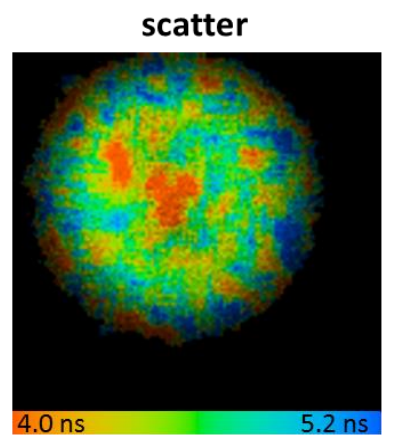

B

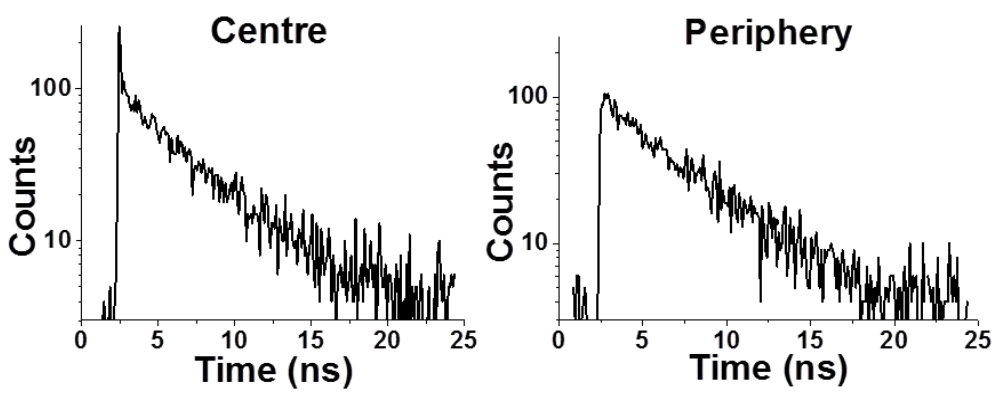

Lifetime with floating

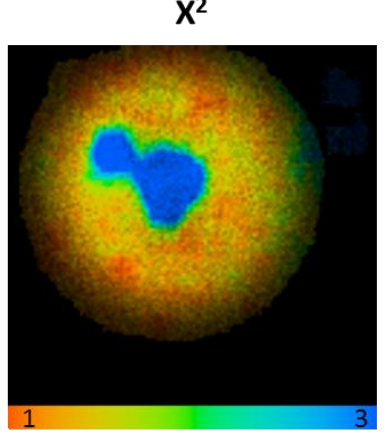
scatter

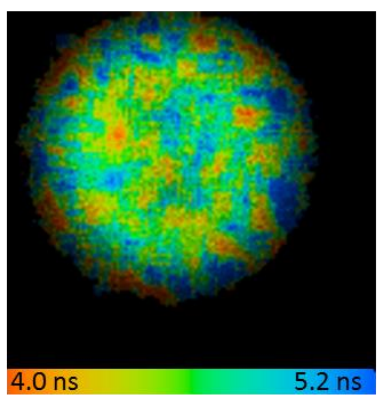

C

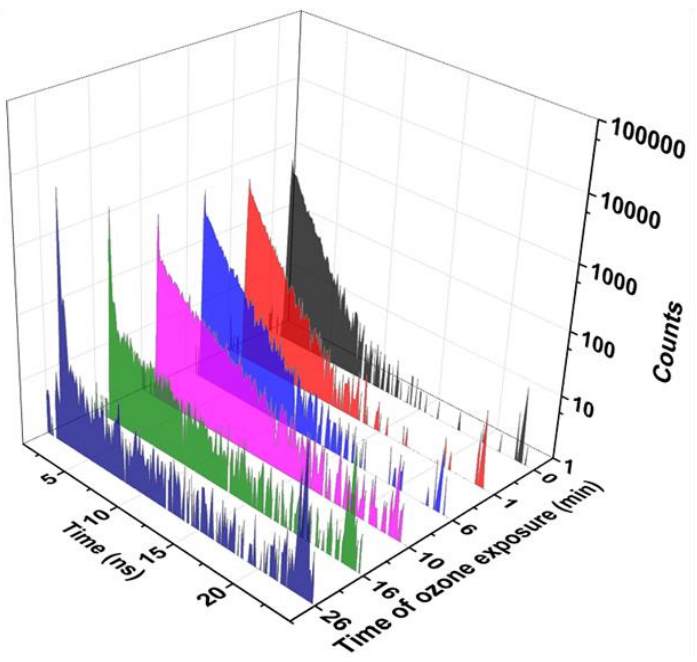

Figure 3: (A) FLIM of a $5 \mu \mathrm{m}$ trapped squalene droplet after $30 \mathrm{~min}$ of oxidation with 53ppm ozone. The intensity, lifetime and goodness of fit ( $\chi^{2}$ ) images are shown for a fitting procedure with fixed zero scatter. A lifetime image fitted with floating scatter is also shown, indicating that the longer lifetime attributed to Bodipy- $C_{10}$ remained uniform throughout the particle. (B) Fluorescence decay traces recorded from the centre and the periphery of the droplet shown in (A); (C) Fluorescence decay traces recorded from whole droplet over the course of ozonolysis; it could be seen that a scattering peak becomes more prominent as ozonolysis progresses. 

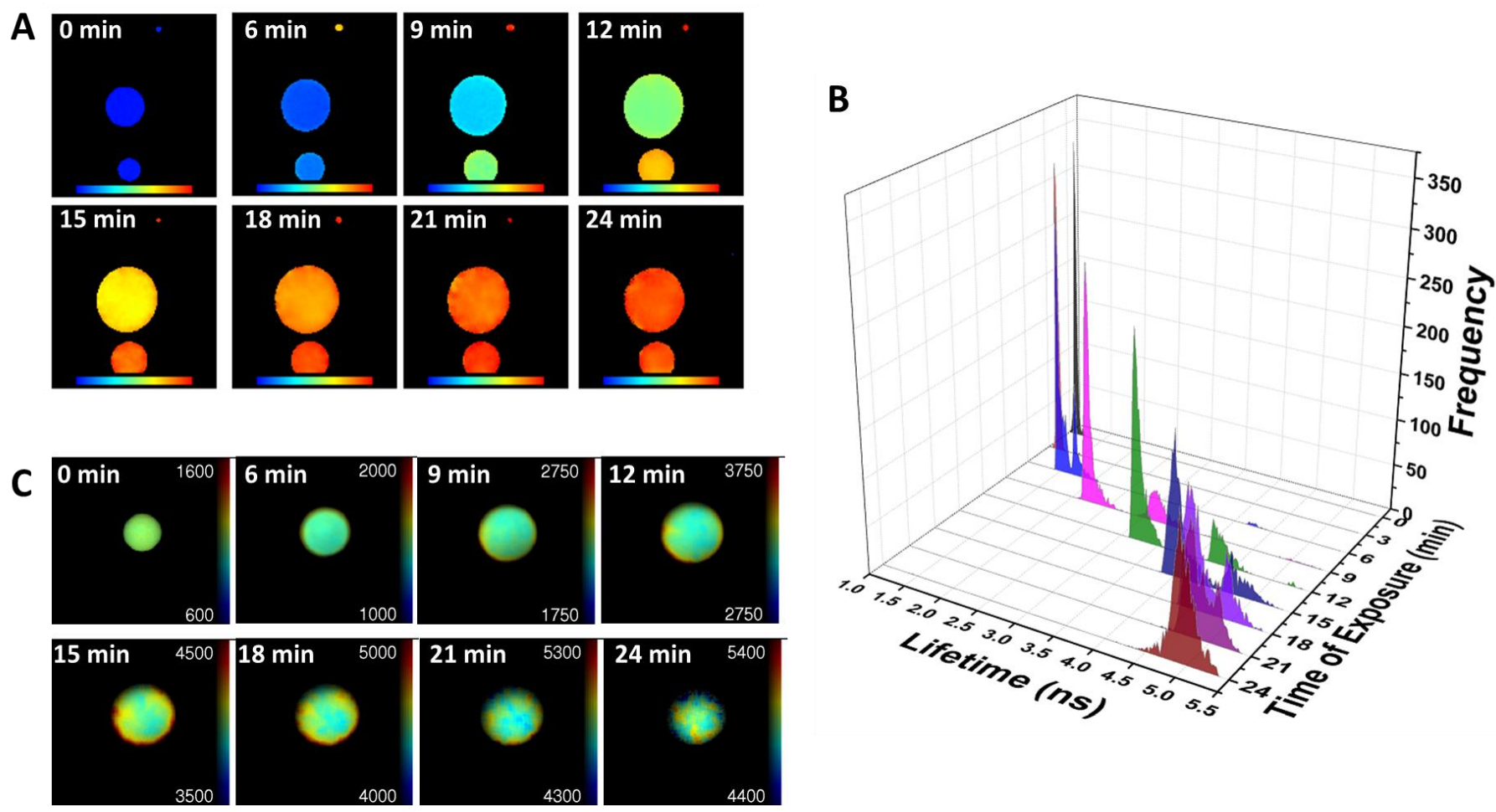

Figure 4: (A) FLIM of squalene oxidation on the coverslip as a function of a droplet diameter, for 11, 35 and $62 \mu \mathrm{m}$ droplets between 0 min and 24 min oxidation time. The lifetime colour hue is between 1.0-5.4 ns for all images (B) 3D lifetime histogram plot depicting the change of lifetime during oxidation of squalene droplets shown in (A). (C) Monitoring heterogeneity over the course of ozonolysis in the $62 \mu \mathrm{m}$ droplet. Each FLIM image is plotted using a rainbow colour hue of 1 ns width around its average lifetime. Thus the progressive spread of colour indicates the widening of the lifetime histogram and hence increased heterogeneity.

In spite of deviations from true aerosol phase, measuring aerosol droplets deposited on the coverslip offers several advantages. Namely (i) larger droplets can be studied allowing to better observe possible heterogeneities in the oxidised droplet composition and (ii) there is an opportunity for simultaneous measurement of more than one droplet under exactly the same experimental conditions and hence the possibility of better counting statistics. The latter advantage is depicted in Figure 4 where three droplets of different sizes (11, 35 and $62 \mu \mathrm{m}$ ) are being imaged simultaneously while reacting with ozone. As seen in Figure $4 \mathrm{~B}$ the total time required for all three droplets to reach at their maximum lifetime is 21 minutes, however individual droplets reach this maximum value at different times: $9 \mathrm{~min}$ for $11 \mu \mathrm{m}$ droplet, 15 minutes for $35 \mu \mathrm{m}$ droplet and $21 \mathrm{~min}$ for the largest $62 \mu \mathrm{m}$ droplet. These changes can also be observed on the evolution of the lifetime histogram during oxidation, Figure 4B. At the start of the oxidation all droplets display an identical lifetime distribution with a sharp and narrow peak at $1.1 \mathrm{~ns}$. During the course of the oxidation, the histograms corresponding to individual droplets separate and widen. Increased histogram width indicates that the oxidation is accompanied by a significant increase in heterogeneity within droplets. This is demonstrated in Figure $4 \mathrm{C}$ for the $62 \mu \mathrm{m}$ droplet, by maintaining the width of the histogram colour hue, while the centre lifetime of the histogram increases in the course of the oxidation. It is clear to see that the spread in colour, therefore heterogeneities in viscosity, increases significantly during oxidation. A possible explanation for this is that the non- volatile ozonolysis products (i.e. the heavier and/or more highly oxidised products) have diffused into different areas of the droplet, resulting in domains of higher viscosity. The aerosol has therefore changed from homogeneous to heterogeneous over the course of oxidation. This observation of increased heterogeneity within oxidised droplets closely mirrors our recent data on ozonolysis of oleic acid droplets deposited on the coverslip ${ }^{17}$. In these oxidised oleic acid droplets we also observed a significant widening of the lifetime histogram of embedded Bodipy- $\mathrm{C}_{10}$ during ozonolysis, more significant than could be expected from the lifetime increase alone, from $1.3 \pm 0.1 \mathrm{~ns}( \pm 7.7 \%)$ to $4.2 \pm 0.6 \mathrm{~ns}( \pm$ 14.5\%). Taken together this data indicates that the liquid-liquid domain separation thought responsible for these microheterogeneities might be a common feature of ozonolysis of unsaturated organics that form aerosol droplets.

We did not detect the glassy core in the coverslip deposited squalene droplets at the end of oxidation. However, we observed evidence of significant spatial heterogeneities resulting from squalene oxidation, Figure 5. In particular, in the larger droplet the lifetime increased first on the surface and then on the inner centre of the droplet suggesting that a viscous front forms at the beginning of oxidation as the oxidants diffuse through the droplet from its surface.

This observation seems to be in contrast to that observed in a levitated squalene droplet, where a more viscous core was surrounded by a less viscous periphery. In order to further assess the physical properties of the oxidised squalene aerosol, we have nebulised, trapped and oxidised squalene droplets 


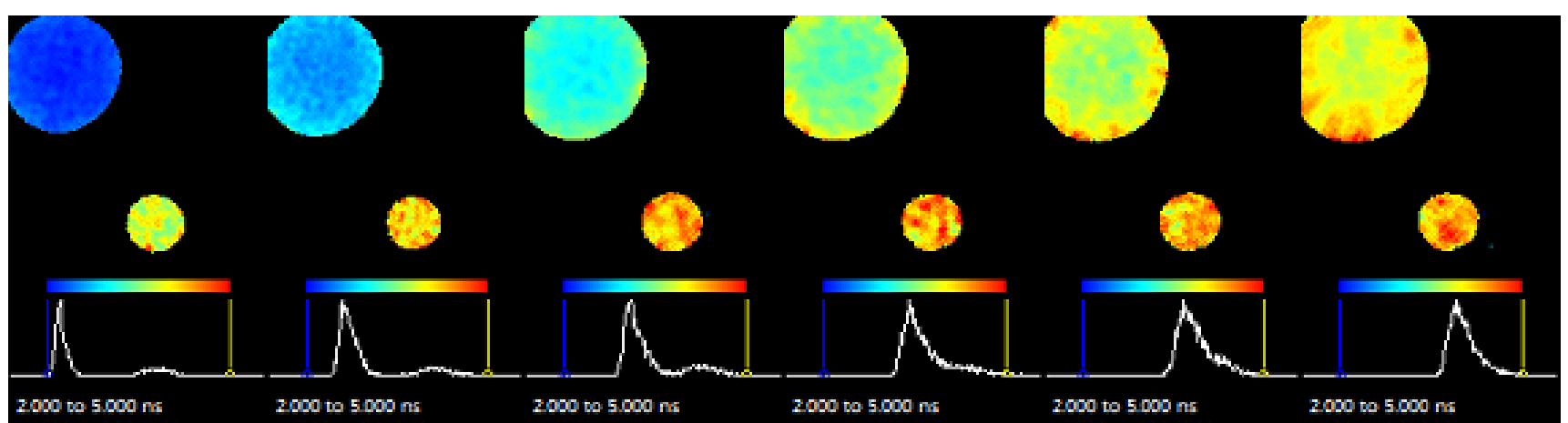

Figure 5: FLIM of squalene droplets (42 and $96 \mu \mathrm{m}$ ) during 53 ppm ozonolysis. The reaction times were 16, 20, 23, 27, 30 and 40 min for images shown from left to right. An uneven increase in a larger particle viscosity (more viscous on the outside, less viscous inside, according to the lifetime colour hue) provides evidence for the formation of a viscous front.

without the prior incorporation of Bodipy- $\mathrm{C}_{10} .30$ minutes after oxidation the rotor was introduced via nebulisation of a Bodipy- $\mathrm{C}_{10}$ methanol solution into the chamber; the fluorescent dye was delivered via multiple collisions with the oxidised and still trapped aerosol droplets. Consequently we have recorded intensity and lifetime images of these droplets, Figure 6 . As can be clearly seen on the intensity image of Figure 6 the fluorescent molecular rotor formed a halo around the outside of the droplet, failing to penetrate into the centre, presumably due to a very high viscosity in the droplet centre. This result further confirms our conclusion from the studies described above, that trapped oxidised squalene aerosol droplets essentially form a centre of extremely high viscosity. The differences between viscosity distribution and front formation in trapped and deposited droplets are likely due to a different size of droplets studied in these two cases: very large 10-100 $\mu \mathrm{m}$ droplets deposited on the coverslip and 5-10 $\mu \mathrm{m}$ trapped droplets. Another possible factor is a deviation from spherical symmetry that is present in coverslip deposited droplets that might prevent solidification. Finally, it is possible that in the absence of contact with a surface, smaller, more volatile oxidation products, such as aldehydes, partition more readily into the gas phase from the trapped oxidised particle. This would potentially result in a more viscous aerosol consisting of heavier molecular weight products.

It is noteworthy that the oxidation of squalene droplets on

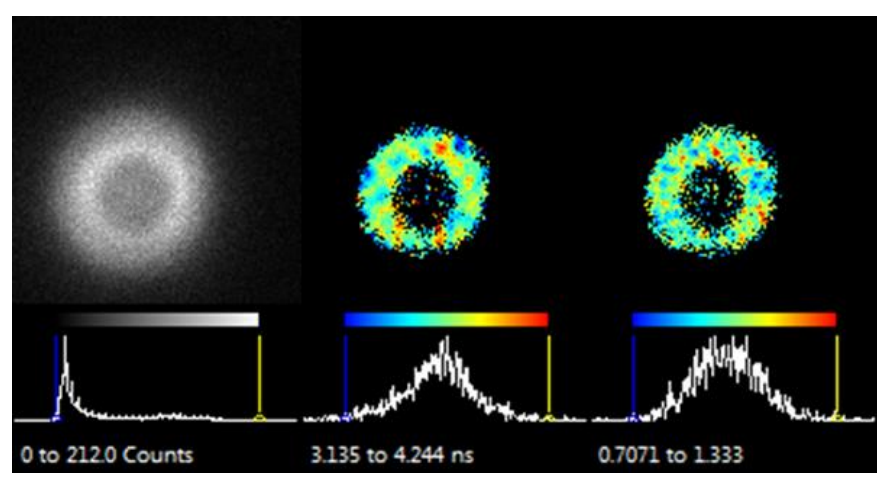

Figure 6: Imaging of a trapped squalene droplet oxidised with $53 \mathrm{ppm}$ ozone for $30 \mathrm{~min}$, Bodipy- $\mathrm{C}_{10}$ was added by nebulisation after the oxidation was complete. Fluorescence intensity (left), lifetime (centre) and goodness of fit (right) images are shown. the coverslip is accompanied by an apparent increase in the droplet size, Figures 4 and 5 , by ca $30 \%$. This size increase can be a consequence of an increased particle mass caused by the uptake of oxygen atoms during the ozonolysis, similar to that recorded during the ozonolysis of arachidonic acid aerosol. ${ }^{39}$ However, it is also possible that this apparent increase is caused by a change in contact angle with the surface, due to the presence of more hydrophilic oxidation products.

To investigate the chemistry of the trapped squalene particle undergoing ozonolysis, a complimentary experiment was performed using a similar optical trap configured with Raman spectroscopic probe (Figure S7, ESI). The normalized spectra reveal the expected chemistry of ozonolysis of $\mathrm{C}=\mathrm{C}$ double bonds, in particular, the fall in the $C=C$ peak at 1620 $\mathrm{cm}^{-1}$ relative to the rest of the signal. The addition of oxygen to the particle can be observed as a generation of carbonyl bonds and peroxide bonds at $1682 \mathrm{~cm}^{-1}$ and $818 \mathrm{~cm}^{-1}$, respectively. There is also the loss of distinction of the $\mathrm{C}-\mathrm{H}$ twisting and wagging modes in the $1200-1400 \mathrm{~cm}^{-1}$ region, although the relative stability of the stretching modes in the fingerprint region shows that the bonds remain intact.

To further assess the evolution and chemical aging of OA in the atmosphere, we have performed preliminary experiments investigating the change in viscosity of squalene aerosol particles upon reaction with hydroxyl radicals $\left({ }^{\circ} \mathrm{OH}\right)$. The $\mathrm{OH}$ radicals were produced by passing $\mathrm{H}_{2} \mathrm{O}_{2}$ vapour through a flow tube exposed to UV light ( $\mathrm{N}_{2}$ was a carrier gas). We have confirmed that the hydroxyl radicals are, indeed, produced by the photolysis of $\mathrm{H}_{2} \mathrm{O}_{2}$ by flowing the resulting gas through the solution of 3'-(p-hydroxyphenyl) fluorescein (HPF) in water $(5 \mu \mathrm{M})$ and observing a steady increase in fluorescence as fluorescein is being formed (Figures S8-9, ESI). This is expected upon HPF oxidation by hydroxyl radicals. ${ }^{40}$ The resulting hydroxyl radicals ${ }^{41}$ were used to oxidise squalene droplets, both on the coverslip and in the optical trap. While it was not possible to determine the concentration of hydroxyl radicals in our setup, we ensured that ${ }^{\circ} \mathrm{OH}$-induced oxidation has resulted in an asymptotic value for the final viscosity, i.e. the reaction was carried out to completion and we were able to compare the endpoints of ${ }^{\circ} \mathrm{OH}$ and $\mathrm{O}_{3}$ oxidation reactions.

Hydroxyl radicals are short lived and highly reactive oxidants eventually reacting not only with squalene and its 
products but also with Bodipy- $\mathrm{C}_{10}$. We observed a steady increase in fluorescence lifetime from 1.1 ns to 3.0 ns during oxidation (Figure S10, ESI), which was accompanied by a steady decrease in the fluorescence intensity of the rotor, consistent with its bleaching. As a result, the viscosity of squalene droplets reaches the measured maximum value of 350-500 mPa s only, corresponding to the measured lifetime of molecular rotor of 2.6-3.0 ns.

In the current experimental set up $\cdot \mathrm{OH}$ oxidation of squalene resulted in a lower degree of oxidation of the reaction products, leading to the smaller increase in viscosity compared to ozonolysis, which is clearly visible in the high resolution mass spectra obtained from droplets oxidised with ozone and $\cdot \mathrm{OH}$ (Figure S11, ESI). It is possible that ${ }^{\circ} \mathrm{OH}$ effectively cleaves oligomerisation products, since it is a strong oxidant and is capable of hydrogen abstraction, ${ }^{42}$ thus resulting in lower viscosity. It is likely that the final viscosity could be higher in an air-saturated environment (rather than in $\mathrm{N}_{2}$ as in our case) due to the possibility of more oxidising chemistry.

The droplets containing bleached Bodipy- $\mathrm{C}_{10}$ were left to react for more than 2 hours and then released on the coverslip in order to study their morphology. The observed brightfield images (Figure S12, ESI) revealed that the droplets were still liquid, thus concluding that viscosity did not increase as dramatically as was the case with the ozone oxidation.

\section{Conclusions}

We quantitatively measured the microviscosity changes occurring in squalene aerosol droplets in the course of chemical aging using either ozone or hydroxyl radicals as oxidants. The use of the Bodipy- $\mathrm{C}_{10}$ molecular rotor that was fully incorporated in the liquid phase(s) of the droplets allowed us to record the microviscosity changes with unprecedented temporal and spatial resolution. We were also able to compare the effect of oxidation on the viscosity of the droplets deposited on the coverslip surface and those levitated via optical tweezers, i.e that represented a closer match to the true atmospheric aerosol phase. The experiments on the trapped droplets and on the coverslip showed similar oxidation kinetics, end point lifetimes and lifetime histogram spreads.

We found that complete ozonolysis of a trapped squalene droplet resulted in a partial solidification of the particle, producing a highly scattering inner core. By contrast, the droplets deposited on the coverslip showed the formation of the viscous front on the outside of the particle. There could be several reasons for these differences: (i) different size of droplets studied in these two cases, (ii) a deviation from spherical symmetry that is present in coverslip deposited droplets that might prevent solidification and (iii) easier volatilisation of products in the absence of coverslip contact. These factors would potentially result in a more viscous aerosol consisting of heavier molecular weight products for the trapped particles.
In both setups we observed large heterogeneities in the final oxidised particles that could be indicative of liquid-liquid phase separated domains that exist as an intermediate step in the course of solidification.

We believe that our study provides a unique tool to study a variety of complex aerosols in their true aerosol phase. The microscopic viscosity values that are revealed by this study should allow further improvements in our understanding of the chemical aging of organic aerosols through suitable modelling and experimentation.

\section{Experimental}

\section{Materials}

The molecular rotor used was meso-alkoxyphenyl-4,40-difluoro-4-bora-3a,4a-diaza-s-indacene (Bodipy- $\mathrm{C}_{10}$ ), synthesised as described previously ${ }^{43}$. All solvents used were of spectroscopic grade. Stock solutions of Bodipy- $C_{10}$ were prepared at a concentration of $2 \mathrm{mM}$ in squalene or methanol as required. Squalene was purchased from Sigma-Aldrich in liquid form, $>98 \%$ purity.

\section{Aerosol preparation}

Squalene or squalane was mixed with Isopropanol at a ratio of $1: 15$. Bodipy- $C_{10}$ was added from a stock solution to produce a final concentration of $20 \mu \mathrm{M}$. Aerosol was produced by nebulisation of the mixture via the Electronic Omron MicroAir U22 Mesh Nebuliser. Alternatively, the mixture of squalene and isopropanol 1:15 was nebulised without Bodipy- $\mathrm{C}_{10}$ and the dye was later added to trapped droplets via nebulisation of Bodipy- $\mathrm{C}_{10}$ solution in methanol at $20 \mu \mathrm{M}$.

\section{Environmental chamber and gas flow}

The chamber was made of aluminium with dimensions of $10 x$ $10 \times 1 \mathrm{~cm}$. The influx and outflux ports were placed on the same side to ensure a smooth gas flow and minimal droplet displacement. On the top and bottom sides of the chamber two windows, one on each side, of borosilicate glass coverslips ( $0.15 \mathrm{~mm}$ thickness) allowed transmission of the trapping beams in the environmental cell. High vacuum grease (Dow Corning) was used to attach the coverslips to the chamber and to avoid gas leakage from the surface in-between. A digital humidity and temperature sensor (SHT-75, Sensirion) was inserted into the chamber via a dedicated small inlet. The gas flow was regulated by the mass flow controller (MKS, 0-500 sccm). Ozone was generated by passing industrial air through a photolysis tube with a $254 \mathrm{~nm}$ UV light source (Pen-Ray mercury lamp, 90-0004-07). The lamp had a controllable ozone concentration capability of 1-1000 ppmv. An ozone concentration of $53 \mathrm{ppm}$ was used for all experiments as measured by a Thermo Scientific $\mathrm{O} 3$ analyser - model 49i.

Hydroxyl radicals were produced by bubbling nitrogen gas though hydrogen peroxide solution heated to $60{ }^{\circ} \mathrm{C}$. The resulting $\mathrm{H}_{2} \mathrm{O}_{2}$ vapour was passed through a flow tube exposed to the fully uncovered UV light source (Pen-Ray mercury lamp, 
90-0004-07). The production of $\cdot \mathrm{OH}$ was verified by increased fluorescence of hydroxyphenyl fluorescein (HPF), a hydroxyl radical sensor purchased from Thermo Fisher Scientific. It was considered that during the oxidation experiments the UV lamp might not convert all of the oxygen into ozone or $\mathrm{H}_{2} \mathrm{O}_{2}$ into $\cdot \mathrm{OH}$. These gas-phase species may cause a background change in fluorescence lifetime. Therefore two controls were carried out to observe the effect of oxygen and $\mathrm{H}_{2} \mathrm{O}_{2}$ vapour on the fluorescence lifetime of the trapped squalene/Bodipy- $\mathrm{C}_{10}$ aerosol, by repeating the experiments with the lamp off (ESI, Figure S13).

\section{Optical Trapping}

The aerosol droplets were levitated using two counterpropagating beams of $1064 \mathrm{~nm}$ wavelength. ${ }^{44} \mathrm{~A} \mathrm{Nd}$ :Yag CW IR laser (Ventus 1064, Laser Quantum) was used as a laser source. The trapping beam was split using a beam splitter and the two resulting beams were passed into optical fibres. The beams were expanded and collimated to slightly overfill the apertures of the objectives lenses. The beams were passed from a top objective lens (x50 N.A. 0.65 NIR MPlan Apo, Mitutoyo) and an objective lens (x60 N.A. 1.2 Nikon Apochromat) of the inverted scanning confocal microscope Nikon Eclipse Ti. An asymmetric power balance, of 12 and 9.5 $\mathrm{mW}$ from the top and lower objective lens respectively, was required to effectively trap the droplets. The focal points of the two objective lenses were set at $40 \mu \mathrm{m}$ above the coverslip surface with $5 \mu \mathrm{m}$ separation. The distances were measured though the microscope focus adjustment controlling the movement of the lower microscope objective lens on the $z$ axis. The heating effect from the NIR laser was negligible as the absorbance coefficient of $1064 \mathrm{~nm}$ light in squalene is almost zero (Figure S14, ESI). The inverted scanning confocal microscope Nikon Eclipse Ti used for FLIM was also coupled with a CCD camera, which enabled the imaging of the trapped droplet using brightfield microscopy.

\section{FLIM measurement and lifetime analysis}

All coverslip experiments were performed on an inverted scanning confocal microscope, SP5 II, Leica Microsystems Ltd, with an 663 N.A. 1.2 HCX PL APO CS water immersion objective lens with correction collar (11506279, Leica Microsystems Ltd.) Brightfield and intensity imaging was performed using an internal laser source at $488 \mathrm{~nm}$. Droplet sizes were measured using the Leica LAS software (Leica Microsystems).

The time resolved images were recorded using a time correlated single-photon counting (TCSPC) module, the SPC830, Becker \& Hickl GmbH, using Ti-Sapphire as an excitation source (680-1080 nm, $140 \mathrm{fs}, 80 \mathrm{MHz}$, Vision II, Coherent Inc.). Bodipy- $\mathrm{C}_{10}$ was excited using two photon excitation at $900 \mathrm{~nm}$ and its emission was collected using a 500-650 $\mathrm{nm}$ detection window. The axial point spread function in these measurements was ca $0.8 \mu \mathrm{m}, 45$ thus the images were collected in well-defined slices through the droplet volume. Laser power was less than $200 \mathrm{~mW}$ for all experiments before entering the microscope. The image format was $128 \times 128$ with 12.5 ns acquisition time window of 256 channels. Lifetime image analysis was performed using Becker \& Hickl software SPCImage, TRI2 (Version 2.7, Gray Institute for Radiation Oncology and Biology) and FLIMfit (OMERO).

The FLIM setup used in combination with the optical trapping was inverted scanning confocal microscope Nikon Eclipse $\mathrm{Ti}$ with $x 60$ N.A. 1.2 Nikon apochromat objective lens. The NKT SuperK EXTREME supercontinuum laser was used as source for single photon excitation. The detector used for TCSPC was a cooled high speed PMT: PMC-100-1, Becker-Hickl GmbH.

The instrument response function (IRF) was recorded using the second harmonic generation signal from dried urea on a borosilicate glass coverslip for two photon excitation. A monoexponential decay model was selected to fit the image in all experiments with acceptable values of chi-square $0.8>\chi_{\mathrm{r}}{ }^{2}$ $<1.2$. Thresholding was performed to remove background noise and binning appropriate to have 100 counts per peak for all pixels within the area of interest. It was observed that the FLIM laser could cause an increase in the fluorescence lifetime of the Bodipy- $\mathrm{C}_{10}$ rotor after long exposure times, therefore care had to be taken so as not to induce an effect by control of the FLIM laser power (discussed in the ESI, Figure S15).

\section{Raman spectroscopy of trapped particles undergoing ozonolysis}

The chemical composition of levitated aerosol particles was monitored using Raman spectroscopy. A $514.5 \mathrm{~nm}$ Ar-ion laser (Innova 300C, Coherent) was focused onto the trapped particle through the lower objective of the optical trap. This light source was used to generate Raman signal which provides compositional information about the probed particle. The Raman signal was directed to a spectrograph (Spectrapro $2500 \mathrm{i}, 1200$ groove blazed at $500 \mathrm{~nm}$ ) and imaged onto a CCD camera (Princeton Instruments, Spec10). For greater details on the optical strategy see Rkiouak et al. 2014 ${ }^{12}$. Squalene particles were introduced into the optical trap and subsequently subjected to ozone (11 ppm) generated by passing synthetic air through a penray lamp.

\section{Acknowledgements}

MKK is thankful to the EPSRC for the Career Acceleration Fellowship (EP/I003983/1). CF thanks NERC for a personal studentship NE/J500070/1. CF and MK were supported by ERC grant 279405. We are thankful to the CLF RAL for a programmed access grant LSF1207 (FLIMOLA).

\section{References}

1 J. H. Seinfeld and S. N. Pandis, Atmospheric chemistry and physics : from air pollution to climate change, John Wiley \& Sons, 2012.

2 A. Nel, Science (80-. )., 2005, 308, 804-805.

3 J. Lewtas, Mutat. Res., 2007, 636, 95-133.

4 A. D. Kappos, P. Bruckmann, T. Eikmann, N. Englert, U. 
Heinrich and P. Höppe, Int. J. Hyg. Environ. Heal., 2004, 207, 399-407. J. H. Kroll and J. H. Seinfeld, Atmos. Environ., 2008, 42, 3593-3624.

6 A. Virtanen, J. Joutsensaari, T. Koop, J. Kannosto, P. YliPirilä, J. Leskinen, J. M. Mäkelä, J. K. Holopainen, U. Pöschl, M. Kulmala, D. R. Worsnop and A. Laaksonen, Nature, 2010, 467, 824-7.

7 L. Renbaum-Wolff, J. W. Grayson, A. P. Bateman, M. Kuwata, M. Sellier, B. J. Murray, J. E. Shilling, S. T. Martin and A. K. Bertram, Proc. Natl. Acad. Sci. U. S. A., 2013, 110, 8014-9. C. Kidd, V. Perraud, L. M. Wingen and B. J. Finlayson-Pitts, Proc. Natl. Acad. Sci. U. S. A., 2014, 111, 7552-7. J. W. Grayson, M. Song, M. Sellier and A. K. Bertram, Atmos. Meas. Tech., 2015, 8, 2463-2472.

10 V. Perraud, E. A. Bruns, M. J. Ezell, S. N. Johnson, Y. Yu, M. L. Alexander, A. Zelenyuk, D. Imre, W. L. Chang, D. Dabdub, J. F. Pankow and B. J. Finlayson-Pitts, Proc. Natl. Acad. Sci. U. S. A., 2012, 109, 2836-41.

11 E. Abramson, D. Imre, J. Beránek, J. Wilson and A. Zelenyuk, Phys. Chem. Chem. Phys., 2013, 15, 2983-91. E. Saukko, H. Kuuluvainen and A. Virtanen, Atmos. Meas. Tech., 2012, 5, 259-265. R. M. Power, S. H. Simpson, J. P. Reid, A. J. Hudsonb, Chem. Sci., 2013, 4, 2597.

14 H. C. Price, B. J. Murray, J. Mattsson, D. O'Sullivan, T. W. Wilson, K. J. Baustian and L. G. Benning, Atmos. Chem. Phys., 2014, 14, 3817-3830. H. C. Price, J. Mattsson, Y. Zhang, A. K. Bertram, J. F. Davies, J. W. Grayson, S. T. Martin, D. O'Sullivan, J. P. Reid, A. M. J. Rickards and B. J. Murray, Chem. Sci., 2015, 6, 4876-4883. M. Shiraiwa, M. Ammann, T. Koop and U. Poschl, Proc. Natl. Acad. Sci. U. S. A., 2011, 108, 11003-11008. N. A. Hosny, C. Fitzgerald, A. Vysniauskas, A. Athanasiadis, T. Berkemeier, N. Uygur, U. Pöschl, M. Shiraiwa, M. Kalberer, F. D. Pope and M. K. Kuimova, Chem. Sci., 2015, 7, 1357-1367. C. Fitzgerald, N. A. Hosny, H. Tong, P. C. Seville, P. J. Gallimore, N. M. Davidson, A. Athanasiadis, S. W. Botchway, A. D. Ward, M. Kalberer, M. K. Kuimova and F. D. Pope, Phys. Chem. Chem. Phys., 2016, 18, 21710-21719. M. K. Kuimova, Phys. Chem. Chem. Phys., 2012, 14, 1267186. Q.-H. Hu, Z.-Q. Xie, X.-M. Wang, H. Kang, Q.-F. He and P. Zhang, Sci. Rep., 2013, 3, 2280. T. E. Graedel, Rev. Geophys., 1979, 17, 937. G. Popjack, J. W. Cornforth, R. H. Cornforth, R. Ryhage and D. S. Goodman, J. Biol. Chem., 1962, 237, 56-61.

J. Sci. Food Agric., 2000, 80, 939-966.
A., 2010, 107, 6568-75.

S. Zhou, M. W. Forbes, Y. Katrib and J. P. D. Abbatt, Environ. Sci. Technol. Lett., 2016, 3, 170-174.
L. Petrick and Y. Dubowski, Indoor Air, 2009, 19, 381-391. C. J. Weschler, S. Langer, A. Fischer, G. Bekö, J. Toftum and G. Clausen, Environ. Sci. Technol., 2011, 45, 3872-3879. J. D. Allan, P. I. Williams, W. T. Morgan, C. L. Martin, M. J. Flynn, J. Lee, E. Nemitz, G. J. Phillips, M. W. Gallagher and H. Coe, Atmos. Chem. Phys., 2010, 10, 647-668.

F. Couvidat, Y. Kim, K. Sartelet, C. Seigneur, N. Marchand and J. Sciare, Atmos. Chem. Phys., 2013, 13, 983-996. C. Wang and M. S. Waring, Atmos. Environ., 2014, 84, 222229.

R. Criegee, Angew. Chemie Int. Ed. English, 1975, 14, 745752.

B. K. Coleman, H. Destaillats, A. T. Hodgson and W. W. Nazaroff, Atmos. Environ., 2008, 42, 642-654.

D. R. Fooshee, P. K. Aiona, A. Laskin, J. Laskin, S. A.

Nizkorodov and P. F. Baldi, Environ. Sci. Technol., 2015, 49, 13304-13.

M. R. Dent, I. López-Duarte, C. J. Dickson, N. D. Geoghegan, J. M. Cooper, I. R. Gould, R. Krams, J. A. Bull, N. J. Brooks and M. K. Kuimova, Phys. Chem. Chem. Phys., 2015, 17, 18393-402.

Y. Wu, M. Štefl, A. Olzyńska, M. Hof, G. Yahioglu, P. Yip, D. R. Casey, O. Ces, J. Humpolíčková and M. K. Kuimova, Phys. Chem. Chem. Phys., 2013, 15, 14986.

N. A. Hosny, G. Mohamedi, P. Rademeyer, J. Owen, Y. Wu, M.-X. Tang, R. J. Eckersley, E. Stride and M. K. Kuimova, Proc. Natl. Acad. Sci. U. S. A., 2013, 110, 9225-30. T. Koop, J. Bookhold, M. Shiraiwa and U. Pöschl, Phys. Chem. Chem. Phys., 2011, 13, 19238-55.

J. W. L. Lee, V. Carrascón, P. J. Gallimore, S. J. Fuller, A. Björkegren, D. R. Spring, F. D. Pope and M. Kalberer, Phys. Chem. Chem. Phys., 2012, 14, 8023-31.

K. Setsukinai, Y. Urano, K. Kakinuma, H. J. Majima and T. Nagano, J. Biol. Chem., 2003, 278, 3170-5.

C. R. Ruehl, T. Nah, G. Isaacman, D. R. Worton, A. W. H. Chan, K. R. Kolesar, C. D. Cappa, A. H. Goldstein and K. R. Wilson, J. Phys. Chem. A, 2013, 117, 3990-4000. W. Lai, C. Li, H. Chen and S. Shaik, Angew. Chem. Int. Ed. Engl., 2012, 51, 5556-78.

J. Levitt, M. Kuimova, G. Yahioglu, P.-H. Chung, K. Suhling and D. Phillips, J. Phys. Chem. C, 2009, 113, 11634-11642. L. Rkiouak, M. J. Tang, J. C. J. Camp, J. McGregor, I. M. Watson, R. a Cox, M. Kalberer, A. D. Ward and F. D. Pope, Phys. Chem. Chem. Phys., 2014, 16, 11426-34. C.-Y. Dong, K. Koenig and P. So, J. Biomed. Opt., 2003, 8, 450. 